\title{
The anticounterfeiter's technological tool kit
}

Anticounterfeiters and fraudulent drug makers are caught in a security arms race. No sooner do the good guys develop a new safeguard than the bad guys discover a workaround. Criminals have learned how to make package-protecting holograms, for example, and they sometimes put small amounts of active ingredients into their pills to fool basic chemical tests. As such, security experts caution that no single anticounterfeiting approach is ever likely to win the war. Nevertheless, various groups have hit upon a range of technological approaches that they believe will give investigators a leg up.

Have lab, will travel If you have ever complained that your lab bench was too small, spare a thought for anticounterfeiters

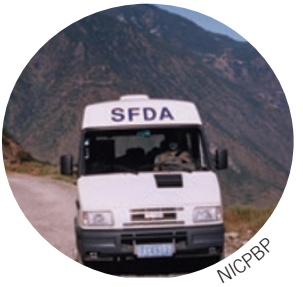
working in mobile

laboratories throughout China. There, twoperson crews drive vans-outfitted with lab equipment rather than back seats-around the country, testing Western and traditional Chinese medicines with near infrared spectroscopy, thin-layer chromatography, simple color tests and microscopes. "The mobile lab is a great design for covering rural areas," says Tom Kubic, president of the Pharmaceutical Security Institute, an anticounterfeiting coalition of drug firms based near Washington, DC. The Chinese National Institute for the Control of Pharmaceutical and Biological Products, which has nearly 400 analytical automobiles, each costing $\$ 130,000$, is currently developing a desktop-sized high-pressure liquid chromatograph to add to the trunk.

\section{Shedding light}

Handheld lab gadgets offer even more portability than anticounterfeiting autos. One of the leading compact

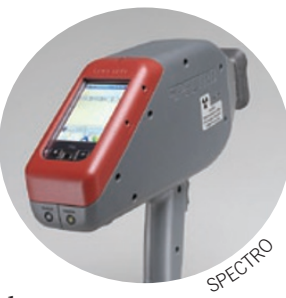
products is the textbook-

sized Raman spectrometer, which shines a laser beam at questionable pills through plastic and blister packs if necessary, and then captures the drugs' unique light-scattering profile. To spot the shams, investigators then compare the optical signature with spectrums stored in a digital reference library. Nigerian drug regulators recently bought Ahura Scientific's TruScan devices for $\$ 50,000$ each, although cheaper devices are available from other manufacturers. "Raman spectroscopy is extremely useful," says Facundo Fernandez, a chemist at Georgia Institute of Technology in Atlanta who tests suspect drugs. "But it is not perfect." Because these spectrometers are susceptible to background interference from inactive components of pills, Fernandez says, groups including his own are working on the next generation of handheld scanners.

\section{Sending out an SMS} Others hope to put anticounterfeiting power directly into the hands of the consumer, with help from a simpler, cheaper

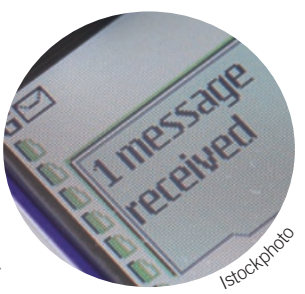
and more ubiquitous

device-the cell phone. In 2008, the Ghanaian nonprofit organization mPedigree asked pill purchasers to text an eight-digit numerical code, hidden under a scratch panel, to a free number that sent back a response, within seconds, to confirm whether the drugs were legitimate or not. Earlier this year, a similar system was also rolled out in Nigeria to prevent the counterfeiting of a diabetes drug, Biofem Pharmaceutical's Glucophage. Simons Bright, co-founder of mPedigree, says he is now working to secure the support of drugmakers and other partners to implement the system more broadly in Nigeria, Rwanda and Kenya.

\section{Nanomarks the spot}

The convoluted supply chains that move drugs from the factory to the pharmacy shelf provide ample opportunity for criminals to sneak their

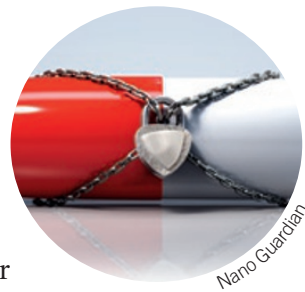
phonies in with the real deals. One solution, some companies believe, is to give each pill its own individual protection. Illinois-based NanoGuardian, for example, has developed a tiny stylus — similar to the sharp tip of an atomic-force microscope-that punches encrypted information into the surface of a drug without adding any additional chemicals. Medicines are then sampled randomly from pharmacies to help inspectors find the fakes. Because legitimate pills can be coded with details about when and where they were made, the nanolabel might also help identify where bogus drugs leak into the supply chain. The capsule-making division of Pfizer recently acquired nonexclusive rights to the nanocodes, but the approach has not yet been rolled out.

Asher Mullard, London

\section{Counting counterfeits}

The number of reported incidences of counterfeit drugs is on the rise. But that's not necessarily bad news - it could be the result of better awareness, notes Tom Kubic, president and CEO of the Pharmaceutical Security Institute, a nonprofit collaboration of 27 pharmaceutical manufacturers based near Washington, DC that has been monitoring the counterfeit situation since 2002. "We're getting closer to understanding the true scope and the full extent of counterfeiting worldwide," he says. Below are the numbers of unique reports of fake drugs that stem from a single organization or product over a seven-year span.

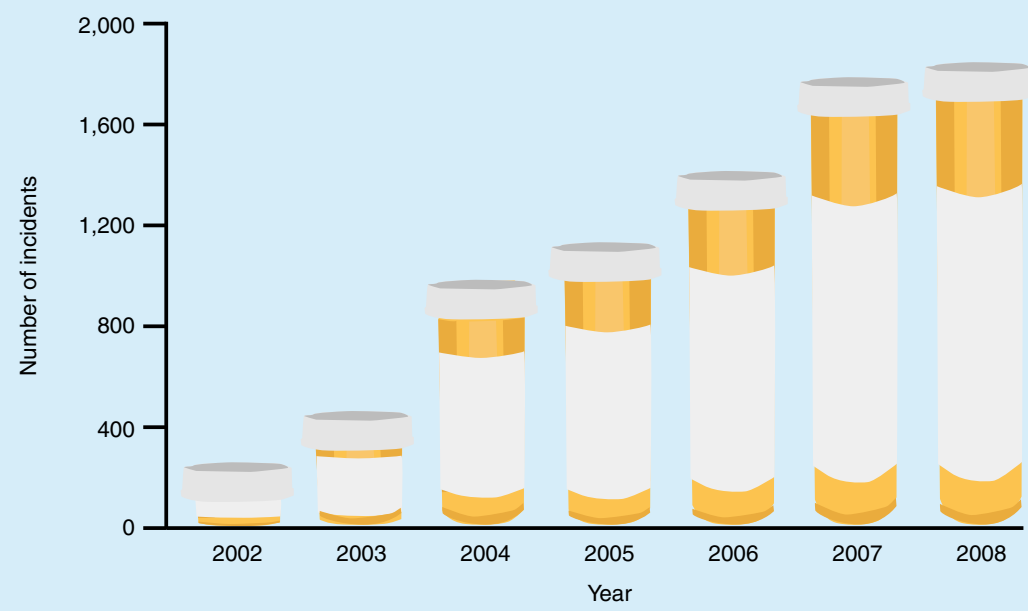

Source: Pharmaceutical Security Institute, Situation Report (2008). 